Chaque seconde compte. Une phrase qui en dit long sur le fait qu'en 2015, les mesures de réanimation initiées par des non-professionnels sont devenues un élément incontournable d'une réanimation réussie dans l'espace public. Un choc doit immédiatement être suivi d'une action, or on en reste trop souvent au choc et le sauvetage n'a pas lieu. Comment faire pour dissiper la crainte de passer à l'action? Cela n'est possible que si l'on y est familiarisé dès le plus jeune âge et que l'on s'y exerce encore et toujours. Les soutiens techniques tels que les défibrillateurs gagnent en importance, mais le sentiment de sécurité qu'ils offrent est un leurre en raison du manque de connaissances et de formation des personnes. Lors d'une intervention ciblée pour sauver une vie, un défibrillateur peut aussi se muer en obstacle si le sauveteur se concentre uniquement sur cet élément. Coordination et formation sont les défis à relever, aujourd'hui comme demain.

\title{
La réanimation par des secouristes volontaires
}

\author{
Patrick Siebenpfund ${ }^{a}$, Gabriela Kaufmann ${ }^{b}$, Roman Burkart ${ }^{c}$, Wolfgang Ummenhofer ${ }^{d}$ \\ a Dr med., spécialiste FMH en anesthésiologie et en médecine intensive, médecin d'urgence SSMUS, Hôpital cantonal de Bâle-Campagne, Bruderholz \\ b Directrice du Secrétariat central SSMUS et SRC \\ c Expert en soins diplômé, collaborateur scientifique auprès de la Fondazione Ticino Cuore \\ d Prof., Département d'anesthésiologie, Hôpital universitaire de Bâle
}

Le Forum du sauvetage de la FMH regroupe l'ensemble des sociétés de discipline médicale impliquées dans les soins d'urgence du lieu de l'événement jusqu'à l'hôpital (= chaîne de sauvetage). En 2010, il s'est exprimé en faveur d'une amélioration de la chaîne de sauvetage dans les «Lignes directrices de la FMH pour le domaine du sauvetage en Suisse». Le présent article traite essentiellement de la réanimation et des cours de Basic Life Support (BLS) effectués par des secouristes volontaires qui devraient dans tous les cas disposer d'une formation de base aux premiers secours.

Lors d'une situation d'urgence dans laquelle le pronostic vital d'une personne est engagé, il s'écoule souvent beaucoup de temps entre le moment où l'alarme est donnée et le moment où les secours arrivent. Or, c'est un fait avéré que le début et la qualité de la réanimation ont un impact durable sur le pronostic du patient [1] tant du point de vue du taux de survie que des éventuels dommages neurologiques consécutifs.

\section{Améliorer les résultats grâce à des cours de réanimation à large échelle}

L'Interassociation de sauvetage (IAS) a calculé le délai d'assistance nécessaire entre le moment où l'alarme est donnée et celui où les secours arrivent auprès du patient, à savoir 15 minutes dans les régions rurales et 10 minutes en ville, et ce dans $90 \%$ des cas. En cas d'arrêt cardio-respiratoire, il est évident que sans réanimation par des secouristes et malgré des délais d'assis- tance courts, les secours professionnels arrivent souvent trop tard. Les données de style Utstein [2] attestent que seuls 6-7\% des patients ayant subi un arrêt cardio-respiratoire survivent (degré de résultat neuro-

Ce n'est que par la mise en œuvre de mesures de réanimation de base immédiates que le patient dispose de réelles chances de survie.

logique 1-2) [3]. Au cours des 30 dernières années, ce taux n'a malheureusement guère pu être amélioré au plan international, sauf exception dans des régions spécifiques, et ce malgré les gros efforts consentis en matière de formation. Or, il est possible d'améliorer grandement ce score grâce aux premiers soins prodigués par des secouristes volontaires, à l'exemple du Tessin qui fait œuvre de pionnier dans ce domaine en Suisse. Par la mise sur pied de programmes de soutien diversifiés pour les sauvetages effectués par des nonprofessionnels, les Tessinois ont pu arriver à un taux de survie exceptionnel de $40 \%$ et visent même les 50\%. Le Swiss Resuscitation Council (SRC) a rédigé des recommandations en matière de réanimation à l'intention du grand public, mais ce sujet n'étant pas jugé suffisamment important pour être traité en priorité dans l'agenda politique, aucun responsable politique ne s'y est attelé. Une formation à large échelle de la population dans les gestes de base de la réanimation devrait cependant être au centre des préoccupations, car cela garantirait qu'en cas d'urgence, une personne formée aux manœuvres de réanimation de base qui se trouverait à proximité de l'incident pourrait immédiatement 


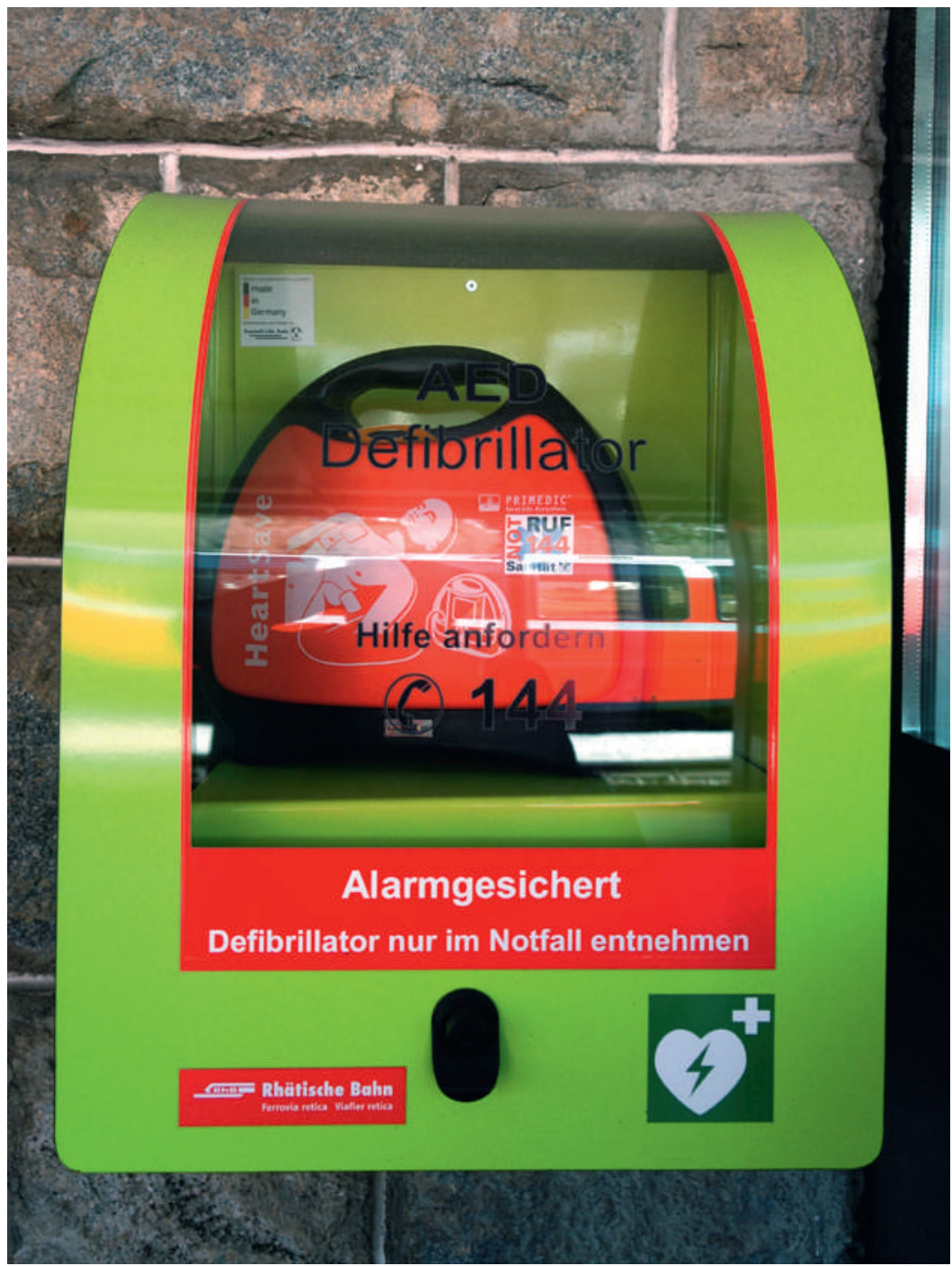

Grâce au défibrillateur externe automatisé installé dans la gare de Saint-Moritz, les gens peuvent sauver des vies. non seulement le système de sauvetage par des secouristes volontaires - qui fonctionne parfaitement dans le sens où la situation est reconnue, l'alarme est donnée et les mesures de réanimation sont immédiatement instaurées par des personnes présentes sur le lieu de l'événement - mais également les instructions au BLS par téléphone par les centrales d'appels sanitaires d'urgence, l'implantation de défibrillateurs automatisés externes dans l'espace public et les systèmes de First Responder. Il est également arrivé que l'arrêt cardio-respiratoire d'une personne connue suscite l'intérêt public pour la question. Mais ce n'est que par la reconnaissance de l'événement et la mise en œuvre de mesures de réanimation de base immédiates que le patient dispose de réelles chances de survie. Il est par conséquent essentiel de former la population à large échelle et de manière efficace au BLS, et ce d'autant plus qu'il est possible aujourd'hui d'acquérir des notions suffisantes en la matière grâce à des directives simplifiées. Cela peut se faire à l'école, comme dans le canton d'Obwald ou encore du Tessin qui forme depuis 6 ans tous les écoliers en fin de scolarité aux techniques BLS. Si l'on compte les 3500 élèves qui terminent chaque année l'école obligatoire, on arrive à un total de 21000 jeunes ayant acquis les aptitudes nécessaires pour reconnaître les situations d'urgence, réagir correctement et transmettre ensuite leurs connaissances à leur famille. Ne vaudrait-il pas la peine de suivre ces exemples et d'intégrer la formation BLS à tous les plans d'études cantonaux? Une telle campagne a d'ailleurs été menée il y plus de 20 ans dans différentes régions des EtatsUnis et notamment à Seattle dans l'Etat de Washington qui a obtenu des résultats exceptionnels en matière de réanimation.

\section{Installer des défibrillateurs externes de manière ciblée et impliquer l'entourage} cours BLS dans les écoles et les entreprises, la mise sur pied de systèmes First Responder [4] et l'instruction par téléphone à l'initiation du BLS par les collaborateurs des centrales d'appels sanitaires d'urgence sont des premiers jalons dans la bonne direction, mais malheureusement seulement au niveau restreint des communes ou des cantons.

\section{Le Tessin montre l'exemple en enseignant la réanimation à l'école obligatoire}

Afin de justifier leur désintérêt pour ce sujet essentiel, les politiques invoquent souvent la question des coûts. Il s'agit là d'une étroitesse d'esprit puisque chaque vie humaine perdue représente une perte de valeur inestimable. C'est à des initiatives individuelles que l'on doit
Les défibrillateurs externes automatisés (DEA) devraient être installés dans des lieux précis où de nombreuses personnes passent, comme les gares, écoles, salles polyvalentes ou bâtiments communaux, et être accessibles au public $24 \mathrm{~h}$ sur 24 . Les DEA publics et privés devraient être recensés et leur emplacement devrait pouvoir être consulté par les centrales d'appels sanitaires d'urgence et par les privés (par ex. via leur smartphone). Il serait également judicieux que le personnel (personnel de surveillance, employés des transports publics, enseignants, concierges, employés communaux, etc.) exerçant dans les lieux publics dotés de DEA soient formés aux manœuvres de réanimation de base et à l'utilisation d'un défibrillateur. En cas d'urgence, les personnes présentes sur le lieu de l'événe- 
ment ou la centrale d'appels sanitaires d'urgence peuvent ainsi rapidement savoir où se trouve le DEA le plus proche. La centrale d'appels sanitaires d'urgence peut ensuite envoyer des personnes formées aux manœuvres BLS sur le lieu de l'incident pour soutenir celles ayant déjà initié les premières mesures de réanimation.

\section{Les DEA devraient être installés dans des lieux précis où de nombreuses personnes passent.}

En revanche, il n'est pas judicieux d'installer des défibrillateurs dans des lieux publics sans concept d'intervention préalable, car leur utilisation n'est efficace que si les manœuvres de réanimation de base sont immédiatement accomplies en parallèle. Les détenteurs actuels et futurs de DEA (communes, entreprises, associations) doivent en tenir compte et être convaincus de l'importance du système BLS/DEA. Des informations sur les directives en matière de réanimation et d'algorithme BLS/DEA sont disponibles sur le site Internet du SRC (www.resuscitation.ch - Directives pour la réanimation 2010).

Pour les régions périphériques situées hors du délai d'assistance fixé par l'IAS, les autorités doivent favoriser et soutenir la création de groupes de First Responder. Il est par ailleurs intéressant de noter que certains cantons (par ex. le Tessin) et certaines villes (par ex. Zurich) ont formé l'ensemble du corps de police aux mesures BLS et muni tous les véhicules de patrouille de défibrillateurs externes et en partie de systèmes d'aide à la respiration artificielle.

\section{Nécessité d'un soutien de la part des médecins et des politiques}

Si les initiatives individuelles ont permis de démontrer que les mesures de premier secours permettaient de sauver des vies, nous avons désormais besoin d'obtenir le soutien des médecins et des politiques et de mener des discussions spécifiques avec les sociétés cantonales de médecine et les médecins cantonaux pour augmenter le taux de survie après un arrêt cardio-respiratoire de manière durable sur l'ensemble du territoire. Les résultats extraordinaires obtenus au Tessin ne sont par ailleurs connus que grâce à la tenue d'un registre cantonal des réanimations qui a été établi conformément aux dispositions légales après examen par la Commission cantonale d'éthique. Si l'on veut en effet pouvoir attester l'efficacité des chaînes de sauvetage, il faut pouvoir se référer à un tel registre, et c'est un principe qui s'applique à l'ensemble de la Suisse. Un registre national des réanimations tel que SwissReCA élaboré selon le modèle tessinois devrait donc être soutenu par le corps médical et les responsables politiques.

Résumé des principes clés:

- Les mesures BLS/DEA prodiguées des secouristes volontaires permettent nettement d'améliorer le taux de survie après un arrêt cardio-respiratoire.

- La formation BLS/DEA doit être intégrée dans les plans d'études scolaires.

- La seule implantation de DEA ne suffit pas. Ceux-ci doivent être intégrés dans un concept d'utilisation pertinent et les groupes cibles à proximité des DEA doivent être formés en conséquence.

- La saisie systématique et la déclaration de tous les DEA publics, semi-publics et privés est la condition préalable à leur utilisation ciblée par la centrale d'appels sanitaires d'urgence et les personnes privées.

- Les systèmes de First Responder doivent être instaurés et soutenus dans les régions appropriées.

- La mise sur pied d'un registre des réanimations pour l'ensemble de la Suisse est judicieuse et doit être soutenue par les médecins et les politiques.

\section{Références}

1 Par souci de lisibilité, seule la forme masculine est utilisée. Nous remercions les lectrices pour leur compréhension.

2 Le rapport style d'Utstein est une notice explicative pour une do cumentation uniforme de l'arrêt cardio-respiratoire.

Une rencontre internationale des organisations impliquées dans la recherche en réanimation a eu lieu en 1990 dans l'abbaye d'Utstein en Norvège. Un groupe de travail, composé de membres de l'American Heart Association, du European Resuscitation Coun cil, de la Heart and Stroke Foundation of Canada ainsi que du Australian Resuscitation Council, a élaboré des recommandations sur la saisie uniforme des données en cas d'arrêt cardio-respiratoire.

3 Drolshagen S. Mit System gegen den Herztod. Rettungsdienst. 2014;37:722-5.

4 First Responder ou "premiers répondants sanitaires»: souvent organisés par groupes et mandatés parallèlement aux services de sauvetage.

En Suisse, ces groupes sont organisés selon un système de milice (à l'instar des pompiers locaux)

Un patient peut ainsi obtenir les premiers soins avant l'arrivée des services de sauvetage. Les First Responder prodiguent les premier soins conformément à leur formation et leur équipement. En Suisse, les systèmes de First Responder ont fait leurs preuves dans les régions à faible densité de population, mais prennent aussi progressivement de l'importance dans les agglomérations. 